\begin{tabular}{|c|c|c|}
\hline $\begin{array}{l}\text { PKS } \\
\text { PUBLIC } \\
\text { KNOWLEDGE } \\
\text { PROJECT }\end{array}$ & $\begin{array}{c}\text { REVISTA DE GEOGRAFIA } \\
\text { (RECIFE) } \\
\text { http://www.revista.ufpe.br/revistageografia }\end{array}$ & $\begin{array}{l}\text { OJS } \\
\begin{array}{l}\text { OPEN } \\
\text { JOUNAL } \\
\text { SYSTEMS }\end{array}\end{array}$ \\
\hline
\end{tabular}

\title{
ESTIMATIVA DOS COMPONENTES DO BALANÇO HÍDRICO NA SUB-BACIA DO RIO ARAPEPÓ COM O MODELO SWAT
}

Joyse Tatiane Souza dos Santos ${ }^{1}$, Adriano Marlisom Leão de Sousa ${ }^{2}$, Hildo Giuseppe Garcia Caldas Nunes ${ }^{3}$, Ana Karla Pontes ${ }^{4}$, Paulo Vinicius Caldas da Silva ${ }^{5}$

\footnotetext{
${ }^{1}$ Programa de Pós-Graduação em Ciências Ambientais-UFPA. Email:joysetaty@hotmail.com

${ }^{2}$ Instituto Socioambiental e dos Recursos Hidricos - UFRA. Email:marlisoms@yahoo.com.br

${ }^{3}$ Programa de Pós-Graduação em Agronomia - UFRA. Email:garibalde13@gmail.com

${ }^{4}$ Programa de Pós-Graduação em Engenharia Ambiental-UFSC.Email:anakarlapontes@hotmail.com

${ }^{5}$ Curso de Engenharia Ambiental e Energias Renováveis - UFRA. Email:paulo_v@hotmail.com
}

Artigo recebido em 28/09/2017 e aceito em 02/11/2018

\begin{abstract}
RESUMO
O objetivo do estudo foi analisar o balanço hídrico da sub-bacia do rio Arapepó, localizada na Vila de Cuiarana, em Salinópolis, no estado do Pará, com o modelo Soil and Water Assessment Tool (SWAT). A análise foi feita através da comparação entre o fluxo do rio simulado pelo modelo e o fluxo do rio observado no local, no período de janeiro de 2008 a abril de 2014, utilizando dados meteorológicos de uma estação micrometeorológica, do Instituto Nacional de Meteorologia (INMET), da Agência Nacional de Águas (ANA) e de dados geocartográficos. Os resultados evidenciaram valores de Coeficiente de Eficiência (COE) de 0,68, um Desvio Padrão (Dv) de 18,68\%, um Coeficiente de Massa Residual (CMR) de 0,2 $\mathrm{m}^{3} \mathrm{~s}^{-1}$ e um Erro Médio (EM) de -0,1 $\mathrm{m}^{3} \mathrm{~s}^{-1}$. Os componentes do ciclo hidrológico gerados pelo modelo mostraram resultados coerentes de quantidade de água no solo (SW), escoamento superficial (SURQ), escoamento subterrâneo (GW_Q) e evapotranspiração (Et). Conclui-se que o uso do modelo em sub-bacias não monitoradas é possível, podendo obter um balanço hídrico bastante coerente e próximo da realidade.
\end{abstract}

Palavras-chave: Bacia de Drenagem; Balanço Hídrico; Modelagem Hidrológica.

\section{ESTIMATION OF THE WATER BALANCE COMPONENTS IN THE ARAPEPÓ RIVER SUB-BASIN WITH THE SWAT MODEL}

\begin{abstract}
This study aimed to analyze the water balance of the Arapepó River sub-basin, located in the village of Cuiarana, Salinópolis, State of Pará, Brazil, using the Soil and Water Assessment Tool (SWAT) model. The analysis was performed by comparing the in site observed river flow and the modeled river flow by the SWAT model, from January 2008 to April 2014. The meteorological data were taken from a micrometeorological station, owned by the National Institute of Meteorology (INMET) and the National Water Agency (ANA), and geocartographic data. Regarding the results, the Efficiency Coefficient (COE) reached values of 0.68, a Standard Deviation (Dv) of $18.68 \%$, a Residual Mass Coefficient (CMR) of $0.2 \mathrm{~m}^{3} \mathrm{~s}^{-1}$ and a Mean Error (MS) of $-0.1 \mathrm{~m}^{3} \mathrm{~s}^{-1}$. The components of the hydrological cycle generated by the model showed coherent results of soil water quantity (SW), surface runoff (SURQ), underground flow (GW_Q) and evapotranspiration (Et). Therefore, the use of the model over unmonitored sub-basins can provide solid data, which in turn can be used to obtain a water balance that is very coherent and close to reality.
\end{abstract}

Keywords: Drainage Basin; Water Balance; Hydrological Modeling. 


\section{INTRODUÇÃO}

O balanço hídrico permite estimar a quantidade de água do movimento de entrada e saída de uma determinada porção de solo, em um período de tempo. As alterações climáticas podem promover inúmeras mudanças na disponibilidade dos recursos hídricos que impactam diretamente no ciclo hidrológico, principalmente na quantidade e qualidade da água que é ofertada (TUNDISI, 2008).

Aplicado em estudos do solo e da água, o balanço hídrico é baseado na relação existente entre a precipitação, a evapotranspiração, o escoamento superficial e o armazenamento da água no solo. A precipitação representa a recarga de água do sistema, enquanto a evapotranspiração representa a combinação entre a evaporação das plantas e a da superfície do solo, estando incluída a transpiração das plantas. Esse processo de evapotranspiração é praticamente o transporte da água de volta para a atmosfera, sendo o inverso do processo da precipitação. O escoamento superficial representa o fluxo superficial da água diretamente na área de interesse. A capacidade de armazenamento representa a quantidade de água que pode ficar retida no solo e nos resíduos sólidos nos casos dos aterros sanitários (FENN et al., 1975; KOMILIS; ATHINIOTOU, 2014).

Muitos estudos têm sido realizados em relação à disponibilidade hídrica de uma bacia através do balanço hídrico, em que a análise das vazões mínimas observadas no período de estiagem, reflete o potencial natural disponível para o abastecimento de água de populações, indústrias, irrigação, navegação, geração de energia elétrica e lançamento de efluentes, entre outros, enquanto que a vazão máxima é importante para o controle de inundações e dimensionamento de obras hidráulicas, entre outros.

Nesse sentido, o SWAT é um modelo hidrológico que permite simular diferentes processos físicos na bacia como, a evapotranspiração, infiltração, escoamento de água, entre outros, com o objetivo de analisar os impactos das alterações no uso do solo sobre o escoamento (superficial e subterrâneo), produção de sedimentos e qualidade de água em bacias hidrográficas não instrumentadas. Com esse modelo é possível simular cenários de uso do solo, permitindo, desta forma, analisar o escoamento de água e a produção de sedimentos de forma distribuída na bacia (NEITSCH et al., 2005).

O SWAT foi desenvolvido para simular os processos que envolvem o uso e a classificação do solo, chuvas e vazão com elevado nível de detalhamento espacial, o modelo permite que a bacia hidrográfica seja dividida em sub-bacias, as quais são subdivididas em 
função de diversas combinações entre características de uso da terra e solo, que são chamadas de unidade de resposta hidrológica (URH, na sigla inglesa HRU) (JIE et al., 2010).

Assim, o artigo tem como objetivo analisar o balanço hídrico da sub-bacia do rio Arapepó e a sua vazão, comparando a vazão simulada (modelo SWAT) com a vazão observada (no local). Após a validação do modelo foi gerado os componentes do ciclo hidrológico da sub-bacia entendendo como são distribuídos na região.

\section{MATERIAL E MÉTODOS}

\section{Área de estudo}

A pesquisa foi realizada na sub-bacia hidrográfica do rio Arapepó, com área de aproximadamente $7,68 \mathrm{~km}^{2}$, localizado na vila de Cuiarana, no município de Salinópolis, na região nordeste do Estado do Pará (Figura 1). A sub-bacia do rio Arapepó tem sua montante próxima a PA-124 e a jusante no rio Arapepó. O solo predominante na bacia é o Gleissolo háplico (50,44\% da área total) e no restante da área é encontrado o Latossolo Amarelo (47,68\% da área), segundo ZEE-PA (2010). A topografia da bacia possui variações entre 4 metros a 40 metros de altitude, em que as maiores altitudes aparecem nas extremidades da sub-bacia, enquanto as de baixa altitude estão por onde passam as redes de drenagem.

Figura 1 - Localização da sub-bacia do rio Arapepó (Salinópolis/PA).

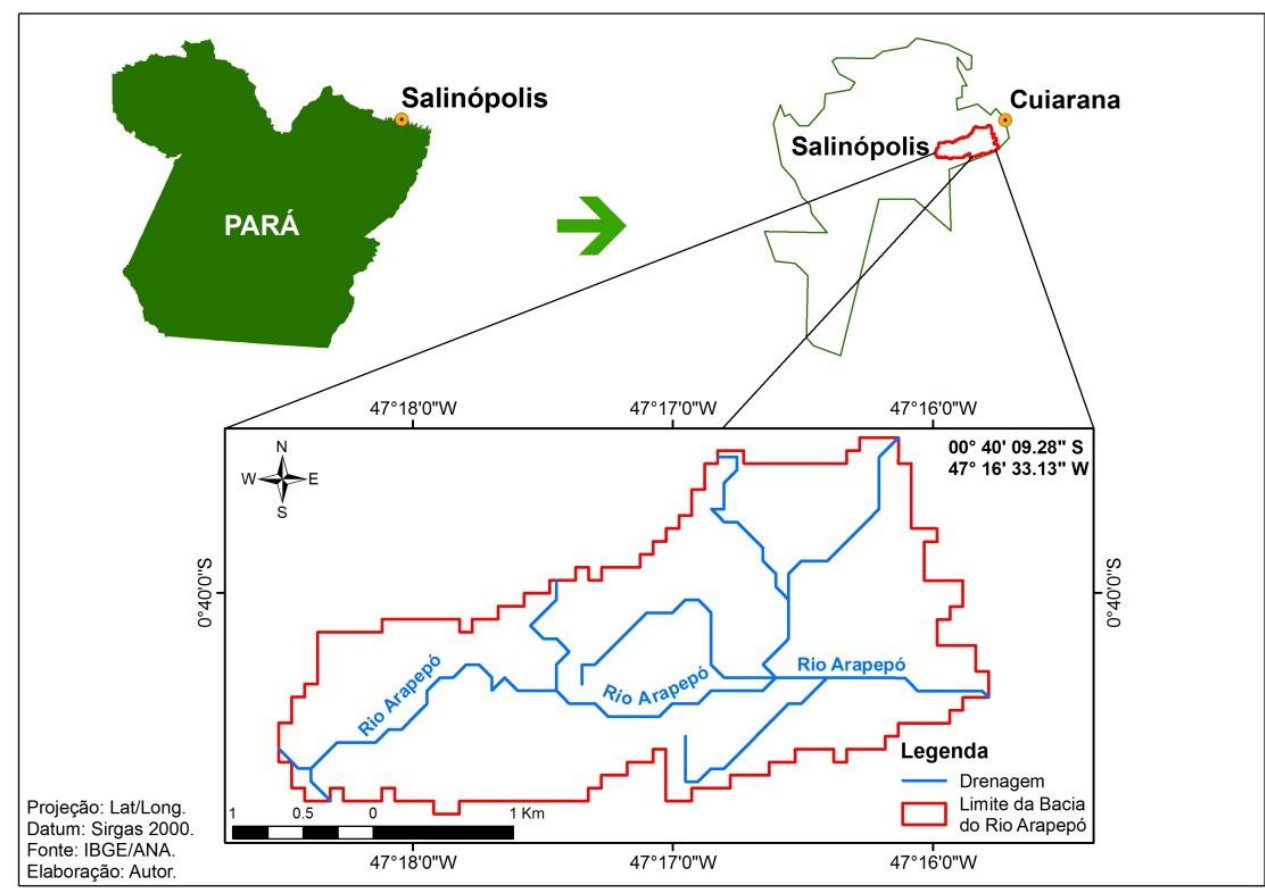

Fonte: Os autores (2018). 
De acordo com a classificação de Koppen, o tipo climático predominante na região é Aw, com reduzida amplitude térmica e pluviosidade anual de $2.100 \mathrm{~mm}$, sendo que $90 \%$ dessa pluviosidade se distribuem nos seis primeiros meses do ano (RODRIGUES et al., 2013). O período chuvoso corresponde aos meses de janeiro a abril e o menos chuvoso de agosto a novembro. A precipitação pluviométrica total anual do período estudado é de 1449,48 mm, com o máximo de chuva recordes em 30 minutos de 930,05 mm. Já a temperatura média das máximas é de $30,98^{\circ} \mathrm{C}$, e das mínimas é de $25,10^{\circ} \mathrm{C}$. A umidade relativa do ar pode variar de 75,0 a $78,0 \%$.

A sub-bacia do rio Arapepó é dominada por áreas Antrópicas Agrícolas que correspondem a 52,32\% de toda a sub-bacia, compreendendo áreas que se encontram ocupadas com culturas agrícolas (temporárias ou permanentes) e silvicultura (reflorestamentos) e da classe de Floresta $(47,68 \%)$ representando todas as formações naturais da floresta, como áreas utilizadas para replantio de espécies florestais para os diversos usos (SANTOS, 2014).

\section{Modelo SWAT}

Para análise do balanço hídrico e a vazão da área de estudo foi utilizado o modelo hidrológico SWAT, associado ao Sistema de Informação Geográfica (SIG) (ArcGis 9.3). O SWAT é um modelo de domínio público e de fonte aberta e integrada que permite ao usuário utilizar conforme a necessidade da pesquisa. De base física e contínua, opera em tempo diário, com o objetivo de predizer o efeito de diferentes cenários de manejo na qualidade da água, produção de sedimentos, bacias hidrográficas e entre outros.

Para a utilização deste modelo hidrológico, foram usados dados de entrada relacionados com as características físicas da área de estudo. Esses dados representam o modelo numérico do terreno, hidrografia, uso e tipo do solo, obtidos a partir do Instituto Brasileiro de Geografia e Estatística (IBGE), Zoneamento Ecológico Econômico do Pará (ZEE-PA) e da Empresa Brasileira de Pesquisa Agropecuária (EMBRAPA), os quais, foram transformados em imagem raster, de resolução espacial de $5 \mathrm{~m}$, na escala de 1:25.000.

Enquanto que os dados climáticos (temperatura máxima e mínima $\left({ }^{\circ} \mathrm{C}\right)$, velocidade do vento $\left(\mathrm{m} \mathrm{s}^{-1}\right)$, umidade relativa $(\%)$, evapotranspiração $(\mathrm{mm})$, precipitação $(\mathrm{mm})$ e radiação solar (cal cm${ }^{-2} \mathrm{dia}^{-1}$ ), foram obtidos a partir de uma estação micrometeorológica, localizada na vila de Cuiarana, além dos dados pluviométricos da Agência Nacional de Águas (ANA) e 
dados meteorológicos do Instituto Nacional de Meteorologia (INMET). A série de dados climáticos utilizados é referente ao período de janeiro de 2008 a abril de 2014.

Neste estudo, o modelo gerou uma sub-bacia com área de 7,68 $\mathrm{km}^{2}$, em que se adicionou manualmente dois pontos de saída, um na sub-bacia 2 e o outro na sub-bacia 3 , gerando desta forma três sub-bacias, onde se considerou apenas a sub-bacia 3 para a discussão e resultados da pesquisa.

Esta subdivisão permite que o modelo identifique diferenças de evapotranspiração para diferentes práticas de agricultura e solos. O escoamento é previsto separadamente para cada URH e posteriormente somado para obter o escoamento total da bacia. Isso aumenta a precisão e dá uma descrição física muito melhor do balanço hídrico para a bacia (NEITSCH et al., 2005). No módulo de hidrologia os principais caminhos que os dados de água percorrem dentro do modelo em cada unidade de reposta são: o escoamento superficial, evapotranspiração, infiltração, percolação, escoamento lateral e águas subterrâneas.

O modelo SWAT se baseia na equação do balanço hídrico (equação 1), o qual, neste momento, processa os resultados em sete módulos: clima, hidrologia, cobertura vegetal/crescimento de plantas, erosão, nutrientes, pesticida e manejo.

$$
S W_{t}=S W_{0}+\sum_{i=1}^{t}\left(R_{i}-Q_{i}-E_{t_{i}}-P_{i}-Q R_{i}\right)
$$

Onde, $S W_{t}$ é a quantidade final de água no solo $(\mathrm{mm}) ; S W_{0}$ é a quantidade inicial de água no solo (mm); t é tempo; $R_{i}$ é a precipitação $(\mathrm{mm}) ; Q_{i}$ é o escoamento superficial $(\mathrm{mm})$; $E_{t_{i}}$ é a evapotranspiração $(\mathrm{mm}) ; P_{i}$ é a percolação $(\mathrm{mm})$ e; $Q R_{i}$ é o fluxo de retorno (é o escoamento de água no interfluxo, sobre a superfície do terreno, após emergir da superfície, ocorrida através da ascensão capilar) (mm).

Portanto, nesse estudo foram analisados os principais componentes do balanço hídrico e que são calculados pelo modelo SWAT, com destaque para: A quantidade total de chuva que precipita sobre a área da sub-bacia durante o tempo de simulação (PRP - precipitação total); Evapotranspiração potencial da bacia (Etp - evapotranspiração potencial); Evapotranspiração real da bacia (Et - evapotranspiração); O volume de água que é propagado pelo canal (WYLD - produção de água total da sub-bacia que alcança o canal); A produção de água que inclui as contribuições do escoamento superficial (SURQ - escoamento superficial); Água que retorna do aquífero raso e entra no canal (GW_Q - escoamento subterrâneo); menos as perdas: Água armazenada no solo nas HRU's (SW - quantidade de água no solo ou PERC - percolação). 
Para a medição dos dados de vazão a observação ocorreu na área da sub-bacia em estudo, no período de 22, 23, 24, 25 e 26 de abril de 2014, com o uso do molinete hidrométrico, através do processo numérico de cálculo de medição convencional de descarga líquida, determinando a velocidade média do fluxo de água que passou pelo ponto de monitoramento, utilizando do método da meia seção. Os valores encontrados foram calculados, através da equação 2:

$$
q_{i}=v_{i} h_{i}\left(\frac{d_{i+1}-d_{i-1}}{2}\right)
$$

Onde, $q_{i}$ é a vazão na seção i $\left(\mathrm{m}^{3} \mathrm{~s}^{-1}\right) ; v_{i}$ é a velocidade média na vertical i $\left(\mathrm{m} \mathrm{s}^{-1}\right) ; h_{i}$ é a profundidade da vertical $\mathrm{i}(\mathrm{m}) ; d_{i+1}$ é a distância a partir do ponto inicial até a vertical posterior (m) e; $d_{i-1}$ a distância a partir do ponto inicial até a vertical anterior (m).

Foi elaborada a análise de sensibilidade automática incorporada ao SWAT, com o objetivo principal identificar quais parâmetros são mais sensíveis quanto ao escoamento superficial, cuja ferramenta dessa análise combina dois métodos: "One-factor-At-a-Time" (OAT) que promove a alteração de apenas um parâmetro a cada simulação e "Latin Hypercube" (LH) que se baseia em simulação de Monte-Carlo, que elimina a necessidade de inúmeras simulações requeridas através de um método de amostragem estratificada e que permite uma estimativa robusta das estatísticas de saída. Tais análises de sensibilidade dos parâmetros são fundamentais para que se saiba onde focar os esforços para a coleta de dados.

Para o desempenho do modelo, esse foi obtido a partir dos valores observados e simulados, calculados com base nos seguintes métodos estatísticos: o coeficiente de eficiência de Nash-Sutcliffe (COE) (varia do $-\infty$ até 1, sendo1 o indicativo de simulação perfeita de evento simulado) (NASH; SUTCLIFFE, 1970), o desvio padrão dos erros (Dv) (quanto mais próximo de $0,00 \%$, mais perfeita é a simulação), o erro médio (EM) (diferença quantitativa entre o evento simulado e o observado) e o coeficiente de massa residual (CMR) (superestima (valores negativos) ou subestima (valores positivos)).

\section{RESULTADOS E DISCUSSÕES}

\section{Análise do desempenho do modelo}

Após a aplicação do modelo para a simulação da vazão na sub-bacia do rio Arapepó ficou evidente que ele pode ser aplicado na estimativa desta variável, pois o SWAT através da inserção das informações da área de estudo conseguiu compreender a sazonalidade da região, 
tanto qualitativamente (análises de eficiência), quanto quantitativamente (superestimou alguns valores de picos de vazão).

$\mathrm{Na}$ simulação inicial, os dados de clima e as características físicas do solo que foram introduzidos no banco de dados do modelo serviram de condições iniciais para que o modelo se adequasse ás características físicas da área de estudo. Os demais parâmetros usados, considerados de base do modelo, foram os de "default". A primeira simulação das vazões diárias e mensais, corresponderam ao período de Janeiro/2008 a Abril/2014, para a sub-bacia 3. Vale ressaltar que para este estudo foram realizadas simulações sem calibração e que foram comparadas com os dados observacionais, medidos na sub-bacia.

Ainda o modelo SWAT foi capaz de reproduzir a vazão simulada utilizando através das condições de base impostas pelo modelo. Comportaram-se de forma adequada as condições climáticas da região, ficando bastante perceptível que ocorreu vazão, apenas quando houve precipitação, os picos de vazões coincidem com os de precipitação, pois no período seco o modelo simula a vazão com tendência a zero ou a valores próximos a zero (Figura 2).

Figura 2 - Vazão diária simulada pelo modelo SWAT com a precipitação para o período de janeiro de 2008 a abril de 2014.

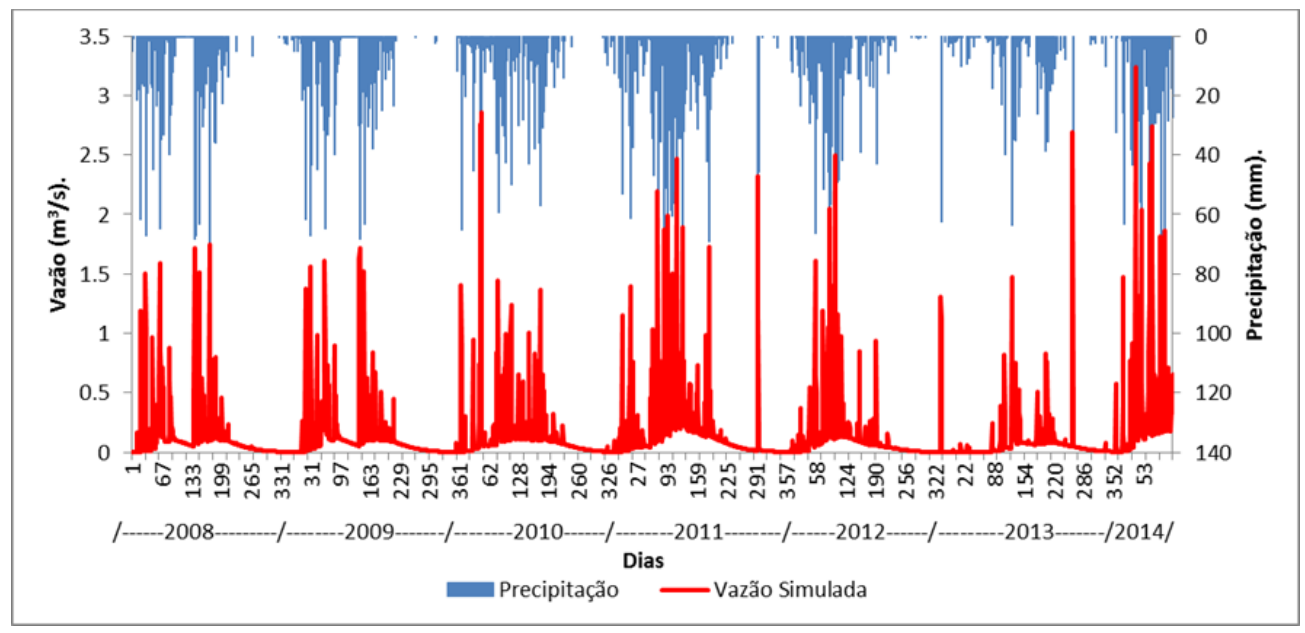

Fonte: Os autores (2018).

No dia 14 de fevereiro de 2010, dia em que ocorreu uma forte chuva com 110,3 mm gerou uma vazão de $2,72 \mathrm{~m}^{3} \mathrm{~s}^{-1}$ mostrando que o modelo é totalmente dependente do mecanismo de regulação do ciclo hidrológico (precipitação). Em períodos em que a precipitação tem valores menores que $10 \mathrm{~mm}$, a vazão tende a zero, como ocorre nos primeiros dias de cada ano, fato este verificado na sub-bacia do rio Arapepó. Alguns picos de vazão precisam ser ajustados em alguns anos (2011, 2012 e 2013), principalmente no período 
seco em que a vazão deveria apresentar baixo ou nenhum valor, fato este que pode estar relacionado pelo modelo não armazenar a água da chuva, gerando altos valores para o período seco (Figura 2). Este tipo de comportamento já foi observado em outros estudos com o modelo SWAT e estes autores destacam algumas dificuldades do modelo em simular o escoamento de base (SOUSA et al., 2015; SANTOS et al., 2018).

A avaliação do modelo para os valores diários obteve bons resultados, um COE de 0,68, mostrando que o modelo teve uma boa eficiência na validação, indicando que ele está validado; um Dv entre 18,68 a 0\%, destacando que o ajuste do evento simulado para a subbacia 3 foi aceitável, quando comparado com outros estudos (WHITE; INDRAJEET, 2005; SOUSA et al., 2015). O CMR igual a $0,2 \mathrm{~m}^{3} \mathrm{~s}^{-1}$ aponta que o modelo está subestimando a vazão observada e o EM, que foi de $-0,1 \mathrm{~m}^{3} \mathrm{~s}^{-1}$, assinalam a subestimativa de alguns valores simulados, com valores quantitativos do total um pouco menores do que realmente são observados. Em geral pode-se comprovar a eficiência do modelo e dizer que o modelo está apto para ser utilizado na sub-bacia do rio Arapepó.

\section{Balanço hídrico}

A PRP evidência o período chuvoso, que vai de janeiro a abril, e o período seco, que vai de agosto a novembro, como foi verificado nas análises da estação de Salinópolis/PA e Cuiarana/PA no inicio do estudo. O mês com maior PRP foi março, com 408,77 mm, e o mês mais seco foi novembro, com 17,25 mm. Possuindo um total acumulado mensal de 1976,43 mm. O mês que teve maior Et para a sub-bacia, foi maio $(21,05 \mathrm{~mm})$, e o mês com o menor valor novembro (11,13 mm). A Et média mensal foi de 16,29 mm (representando 9,89\% da PRP acumulada) (Tabela 1).

A quantidade de água no solo $(\mathrm{SW})(557,71 \mathrm{~mm}$ em média, representando aproximadamente $30 \%$ da PRP acumulada) esteve bem alta, ajudando na regulação da vazão média e na manutenção dos processos fisiológicos. O escoamento superficial (SURQ) foi baixo principalmente no período seco (média de 12,51 mm), e por período chuvoso com média de 167,46 mm em que teve o maior valor no mês de março com 233,49 mm e o menor valor no mês de agosto com 7,25 mm representando 4,45\% da PRP acumulada (Tabela 1). 
Tabela 1 - Principais componentes do balanço hídrico simulado pelo modelo no período de janeiro de 2008 a abril de 2014.

\begin{tabular}{c|c|c|c|c|c|c|c|c|c}
\hline Mês & $\begin{array}{c}\text { SIM } \\
\left(\mathbf{m}^{\mathbf{3}} \mathbf{s}^{-\mathbf{1}}\right)\end{array}$ & $\begin{array}{c}\text { PRP } \\
(\mathbf{m m})\end{array}$ & $\begin{array}{c}\text { SW } \\
(\mathbf{m m})\end{array}$ & $\begin{array}{c}\text { SURQ } \\
(\mathbf{m m})\end{array}$ & $\begin{array}{c}\text { WYLD } \\
(\mathbf{m m})\end{array}$ & $\begin{array}{c}\text { GW_Q } \\
(\mathbf{m m})\end{array}$ & $\begin{array}{c}\text { PERC } \\
(\mathbf{m m})\end{array}$ & $\begin{array}{c}\text { Et } \\
(\mathbf{m m})\end{array}$ & $\begin{array}{c}\text { Etp } \\
(\mathbf{m m})\end{array}$ \\
\hline JAN & 0,10 & 187,31 & 565,47 & 90,59 & 102,56 & 6,00 & 41,23 & 16,24 & 18,43 \\
\hline FEV & 0,23 & 290,99 & 566,12 & 167,85 & 206,26 & 26,28 & 96,04 & 13,39 & 14,12 \\
\hline MAR & 0,32 & 408,77 & 566,22 & 233,49 & 318,70 & 67,63 & 135,40 & 20,30 & 20,56 \\
\hline ABR & 0,30 & 305,26 & 562,94 & 177,91 & 284,22 & 91,78 & 99,66 & 16,88 & 16,94 \\
\hline MAI & 0,25 & 272,07 & 565,64 & 148,73 & 249,99 & 89,72 & 85,80 & 21,05 & 21,51 \\
\hline JUN & 0,20 & 206,57 & 566,01 & 94,49 & 191,10 & 85,26 & 80,89 & 19,01 & 21,45 \\
\hline JUL & 0,15 & 151,80 & 565,23 & 62,55 & 154,66 & 83,10 & 62,12 & 19,49 & 22,52 \\
\hline AGO & 0,07 & 32,73 & 557,92 & 7,65 & 74,29 & 64,17 & 12,09 & 18,39 & 25,18 \\
\hline SET & 0,05 & 20,93 & 547,25 & 15,59 & 50,76 & 34,81 & 2,26 & 13,39 & 25,85 \\
\hline OUT & 0,04 & 28,07 & 541,73 & 19,15 & 36,24 & 16,73 & 1,84 & 12,16 & 27,79 \\
\hline NOV & 0,01 & 17,25 & 539,43 & 7,67 & 14,07 & 6,29 & 0,00 & 11,13 & 24,41 \\
\hline DEZ & 0,03 & 54,68 & 548,57 & 27,79 & 30,65 & 2,08 & 2,82 & 14,02 & 22,27 \\
\hline Média & & & 557,71 & 87,79 & 142,79 & 47,82 & 51,68 & 16,29 & \\
\hline Soma & 1,75 & 1976,43 & & & & & 620,15 & 195,45 & 261,03 \\
\hline
\end{tabular}

Fonte: Os autores (2018).

A sub-bacia produz, em média, $142,79 \mathrm{~mm}$ de água total que alcança o canal (WYLD), sendo sua maior contribuição em março $(318,70 \mathrm{~mm})$ e sua menor contribuição em novembro $(14,07 \mathrm{~mm})$. A produção de água total da sub-bacia que alcança o canal representou 7,22\% do total da PRP acumulada (Tabela 1).

O escoamento subterrâneo (GW_Q) possui uma amplitude de aproximadamente 89,7 $\mathrm{mm}$, escoando subterraneamente mais em abril $(91,78 \mathrm{~mm})$ e menos em dezembro $(2,08 \mathrm{~mm}$ representando 2,42\% da PRP acumulada). A percolação (PERC) esteve alta de janeiro a abril (período chuvoso) e baixa de agosto a novembro (período seco) em virtude da sazonalidade da região (representando 31,37\% da PRP acumulada) possui uma média de 51,68mm, sendo março o mês em que houve maior percolação $(135,40 \mathrm{~mm})$ e outubro aquele em que a percolação foi menor $(1,84 \mathrm{~mm})$, ressaltando que o mês de novembro não houve percolação $(0,00 \mathrm{~mm})$ (Tabela 1$)$.

A evapotranspiração gerada pelo modelo, foi a partir da equação de Penman-Monteith, e que segundo Sousa et al. (2015) principalmente nos períodos secos se mostra deficiente, sugerindo a utilização de outros métodos para o calculo da evapotranspiração, e o mesmo 
utilizou o modelo SEBAL/METRIC para assimilação no modelo SWAT, chegando a valores de eficiência melhores com os valores novos assimilados (COE de 0,89).

Esquematicamente, o balanço hídrico na sub-bacia do rio Arapepó, fica distribuído da seguinte forma: da quantidade de chuva acumulada (1976,43 mm), 28\% ficam armazenadas no solo (SW), 4\% escoam superficialmente (SURQ), $7 \%$ é a água que alcança o canal (WYLD), 3\% escoam subterraneamente (GW_Q), 32\% percolam (PERC), 10\% sofrem evapotranspiração e $13 \%$ evapotranspiração potencial (Figura 3). Vale ressaltar que o modelo gera outros parâmetros como o caso LAT_Q mm que é a contribuição do fluxo lateral da água no interior do perfil do solo, que somado aos parâmetros descritos anteriormente, completando o balanço hídrico.

Figura 3 - Esquema dos componentes do ciclo hidrológico na sub-bacia do rio Arapepó.

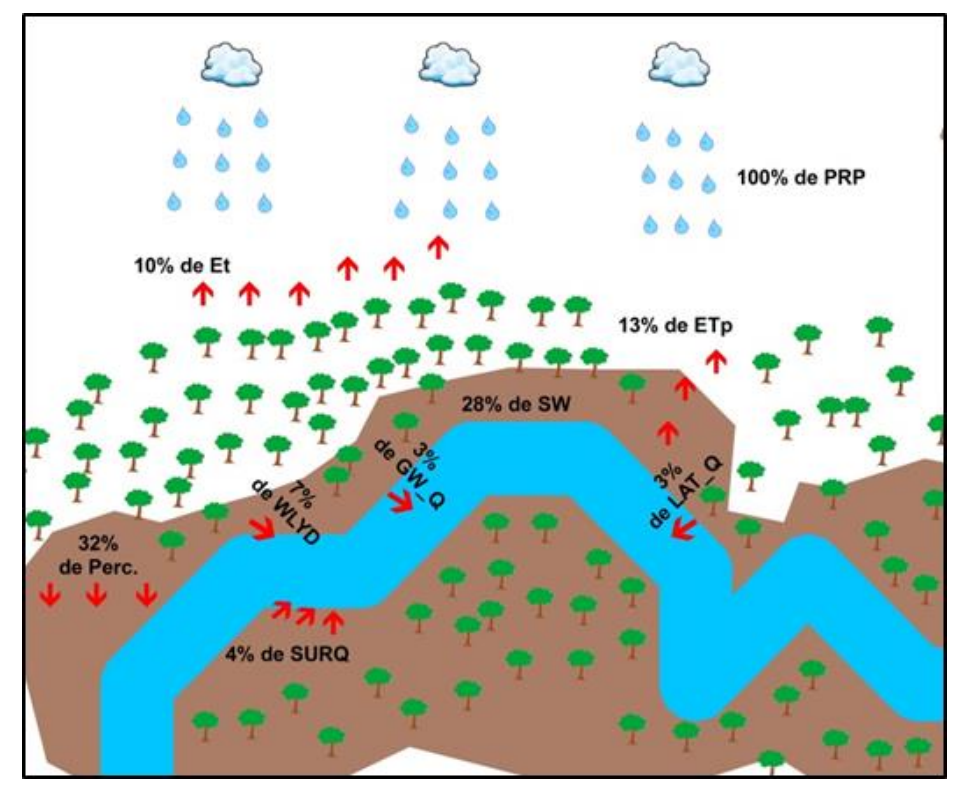

Fonte: Os autores (2018).

\section{Análise de sensibilidade}

No estudo foi elaborada a análise de sensibilidade automática incorporada ao SWAT, identificando-se os parâmetros que o modelo analisa como sendo mais sensíveis na sub-bacia do rio Arapepó, em relação à vazão e que podem ser utilizados como ponto de partida para a calibração do modelo, com o intuito de diminuir possíveis erros. Dos 26 parâmetros identificados destacam-se: o fator do fluxo de base ou constante de recessão (ALPHA_BF); o coeficiente de compensação de evaporação de água no solo (ESCO); a água interceptada (CANMX); o coeficiente de Manning do canal principal (CH_N2); o coeficiente de 
rugosidade do canal principal (CN2); o fator de compensação de absorção pelas plantas (EPCO); o intervalo de tempo para a recarga do aquífero (GW_DELAY); o coeficiente de ascensão de água a zona de saturação (GW_REVAP); o nível limite de água no aquífero livre para ocorrer o fluxo de retorno (GWQMN); a percolação para o aquífero profundo (REVAPMN); o escoamento lateral (LAT_TIME); o coeficiente de percolação da água para o aquífero profundo (RCHRG_DP); a percolação para o aquífero profundo (REVAP_MN); o comprimento de rampa médio para a sub-bacia (SLSUBBASIN); a condutividade hidráulica (SOL_K) e o coeficiente de retardamento do escoamento superficial direto (SURLAG).

O parâmetro cuja sua variação apresentou maior sensibilidade foi o ALPHA_BF. Nele define-se a taxa na qual a água subterrânea retorna ao rio (dias). O segundo parâmetro com maior sensibilidade foi o ESCO, observou-se que pequenas variações no ESCO implicam em alterações no balanço hídrico da bacia. O terceiro parâmetro que chama atenção no rank e é o de maior sensibilidade, foi o GWQMN cuja sua variação afeta o escoamento de base.

\section{Vazão simulada $x$ vazão observada}

O modelo evidenciou uma sazonalidade da vazão positiva, em relação aos picos e as recessões do período estudado, dando destaque para cinco dias, onde o modelo subestimou o pico de vazão no dia $116\left(0,40 \mathrm{~m}^{3} \mathrm{~s}^{-1}\right)$ em relação ao observado $\left(0,60 \mathrm{~m}^{3} \mathrm{~s}^{-1}\right)$, mas pode-se observar que no dia 116 foi um dia de chuva intensa na região em que a sub-bacia está inserida, portanto, o modelo conseguiu acompanhar a sazonalidade deste evento. Assim como ocorreu nos dias 112 e 113. No dia 114 o evento foi simulado perfeitamente, cuja vazão tanto simulada, quanto a observada foi de $0,17 \mathrm{~m}^{3} \mathrm{~s}^{-1}$. Para o dia 115 o modelo superestimou $(0,17$ $\mathrm{m}^{3} \mathrm{~s}^{-1}$ ), enquanto o que o valor observado foi de $0,14 \mathrm{~m}^{3} \mathrm{~s}^{-1}$ (Figura 4).

Em relação ao quantitativo o modelo respondeu a $81 \%$ do total observado, tendo uma subestimativa de 19\% no total de vazão, em alguns dias (112, 113 e 116). Em comparação com a simulação inicial o modelo mostrou boa eficiência qualitativa e quantitativa (Figura 4). 
Figura 4 - Comparação entre as vazões diárias simuladas e observadas com a precipitação, no rio Arapepó.

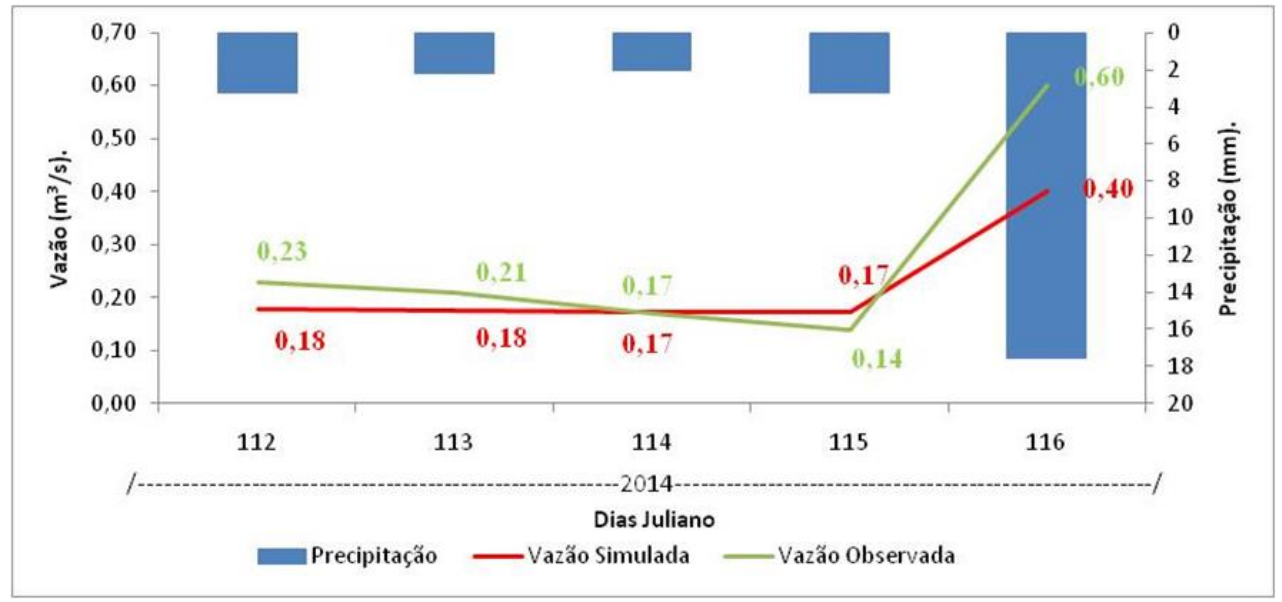

Fonte: Os autores (2018).

Em síntese, os resultados obtidos são coerentes e aceitáveis quando comparados com outros estudos em bacias de mesmo porte. O modelo SWAT já foi aplicado em várias partes do mundo, como no estudo de Van Liew et al. (2003), que aplicaram o modelo SWAT para uma bacia no sudoeste do estado de Oklahoma nos Estados Unidos em períodos distintos e evidenciaram valores de COE distintos em cada período (para o período seco o COE foi de $0,65$ e no período chuvoso foi de 0,45$)$.

Para aplicações do modelo no Brasil, destaca-se: o estudo Sousa et al. (2015), que aplicaram o modelo à sub-bacia do Rio Lajeado, no estado de Tocantins, e obteve resultados satisfatórios na escala mensal, com COE de 0,69, e mostrou ainda que se utilizando a estimativa dos fluxos de calor à superfície a partir de imagens orbitais (através dos modelos SEBAL/METRIC) e aplicando-se estas estimativas no modelo hidrológico SWAT, há melhoras nos resultados mensais. E mais recentemente, Santos et al. (2018), que aplicaram o modelo à bacia do Rio Apeú no nordeste paraense e obtiveram um COE de 0,89.

\section{CONCLUSÕES}

O modelo hidrológico SWAT, largamente utilizado em diversos trabalhos, demonstrou resultados satisfatórios neste estudo, dadas as limitações localizadas na sub-bacia do rio Arapepó, realizando a simulação da série diária de vazão (sub-bacia 3) e na quantificação mensal dos componentes do ciclo hidrológico. Verificou-se que é possível estimar os componentes do ciclo hidrológico através da calibração do modelo SWAT, em especial a vazão, com precisão satisfatória (COE de 0,68) e assim melhor espacializar os dados em 
bacias não monitoradas principalmente. Isso demonstra que o modelo é capaz de simular a vazão na sub-bacia do rio Arapepó, de forma satisfatória.

Em relação ao balanço hídrico as simulações demonstraram que os valores apresentados se confirmam aceitáveis e compatíveis com a realidade apresentada na subbacia. A sub-bacia do rio Arapepó apresenta como característica, o acumulo de água no solo (28\% da PRP acumulada), o que é prescindível para a manutenção dos processos fisiológicos na sub-bacia. Já a percolação que traduz o movimento subterrâneo da água no solo, especialmente nos solos saturados ou próximos da saturação, no Arapepó é influenciado pelo regime de chuva auxiliando neste processo (percola aproximadamente $32 \%$ de água no solo da PRP anual), principalmente no período chuvoso.

Portanto, o uso do modelo SWAT é fundamental para o planejamento e gerenciamento de sub-bacias não monitoradas, o espaço geográfico como um processo de construção e constantes transformações, influência nas dinâmicas fluviais e altera o comportamento natural dos canais, desse modo, os diversos usos do solo, como exemplo o desmatamento, o avanço das áreas urbanas no entorno da bacia, reduz a capacidade de infiltração, aumenta o escoamento superficial, provocando erosões, resultando em assoreamentos do leito e as enchentes da planície de inundação.

\section{REFERÊNCIAS}

FENN, D.; HANLEY, K.; GEARE, T. Use of the water balance Method for predict in leachate generation from solid waste disposal sites. United States: Environmental Protection Agency, 1975. 30 p.

JIE, Z.; GUANG-YONG, L.; ZHEN-ZHONG, H.; GUO-XIA, M. Hydrological cycle simulation of the irrigation district based on a SWAT model. Math Comput Model, New York, v. 51, p. 1312-1318. 2010.

KOMILIS, D.; ATHINIOTOU, A. A water budget model for operating landfills: An application in Greece. Waste Manag Res, London, v. 32, n. 8, p. 717-725. 2014.

NASH, J. E.; SUTCLIFFE, J. V. River flow forecasting through conceptual models. Part I: A discussion of principles. J Hydrol, New York, v. 10, n. 3-4, p. 282-290. 1970.

NEITSCH, S. L. et al. Soil and Water Assessment Tool: Theoretical documentation version 2005. Texas: Backland Research Center, 2005. 460 p. 
RODRIGUES, J. C.; SOUZA, P. J. de O. P. de; LIMA, R. T. de. Estimativa de temperaturas basais e exigência térmica em mangueiras no nordeste do estado do Pará. Rev Bras Frutic, Jaboticabal, v. 35, n. 1, p. 143-150, mar. 2013.

SANTOS, J. T. S. dos. Aplicação do modelo hidrológico SWAT para a sub-bacia do rio Arapepó (Salinópolis/PA). 2014. 84 f. Dissertação (Mestrado em Ciências Florestais) Universidade Federal Rural da Amazônia, Belém, 2014.

SANTOS, J. T. S. dos; NUNES, H. G. G. C.; PONTES, A. K. dos S.; SOUSA, A. M. L. de. O Modelo SWAT como ferramenta para a gestão de recursos hídricos: Um exemplo aplicado no rio Apeú, Castanhal/PA. REGA, Porto Alegre, v. 15, n. 3, p. 1-13. 2018.

SOUSA, A. M. L. de; VITORINO, M. I.; CASTRO, N. M. do S. R.; BOTELHO, M. do N.; SOUZA, P. J. O. P. de. Evapotranspiration from remote sensing to improve the Swat Model in eastern Amazonia. Floresta Ambient, Rio de Janeiro, v. 22, n. 4, p. 456-464. 2015.

TUNDISI, J. G. Recursos hídricos no futuro: problemas e soluções. Estud av, São Paulo, v. 22, n. 63, p. 7-16. 2008.

VAN LIEW, M. W.; JURGEN, G. Hydrologic simulation of the little Washita river experimental watershed using SWAT. J Am Water Resour Assoc, United States, v. 39, n. 2, p. 413-426. 2003.

WHITE, K. L.; INDRAJEET, C. Sensitivity analysis, calibration, and validations for a multisite and multivariable SWAT model. J Am Water Resour Assoc, United States, v. 41, n. 5, p. 1077-1089. 2005.

ZEE-PA/ZONEAMENTO ECOLÓGICO-ECONÔMICO DA ZONA LESTE E CALHA NORTE DO ESTADO DO PARÁ. Diagnóstico do meio Físico-Biótico. 1. ed. Belém: PARÁ RURAL, 2010. 326 p. 